\title{
Review Article \\ Lepton Number Violation and Neutrino Masses in 3-3-1 Models
}

\author{
Richard H. Benavides, ${ }^{1}$ Luis N. Epele, ${ }^{2}$ Huner Fanchiotti, ${ }^{2}$ \\ Carlos García Canal, ${ }^{2}$ and William A. Ponce ${ }^{3}$ \\ ${ }^{1}$ Facultad de Ciencias Exactas y Aplicadas, Instituto Tecnológico Metropolitano, Medellín, Colombia \\ ${ }^{2}$ Laboratorio de Física Teórica, Departamento de Física, Universidad Nacional de La Plata, IFLP, CONICET, \\ C.C. 67-1900 La Plata, Argentina \\ ${ }^{3}$ Instituto de Física, Universidad de Antioquia, A.A. 1226 Medellín, Colombia
}

Correspondence should be addressed to Richard H. Benavides; ribebenavides@gmail.com

Received 21 December 2014; Accepted 24 February 2015

Academic Editor: Niels Bjerrum-Bohr

Copyright (C) 2015 Richard H. Benavides et al. This is an open access article distributed under the Creative Commons Attribution License, which permits unrestricted use, distribution, and reproduction in any medium, provided the original work is properly cited. The publication of this article was funded by SCOAP $^{3}$.

Lepton number violation and its relation to neutrino masses are investigated in several versions of the $S U(3)_{c} \otimes S U(3)_{L} \otimes U(1)_{x}$ model. Spontaneous and explicit violation and conservation of the lepton number are considered. In one of the models (the socalled economical one), the lepton number is spontaneously violated and it is found that the would be Majoron is not present because it is gauged away, providing in this way the longitudinal polarization component to a now massive gauge field.

\section{Introduction}

The colorless and electric neutral charge distinguish the three left handed neutrinos $\nu_{I L}^{0}, l=e, \mu, \tau$ from the other fermions of the $S U(3)_{c} \otimes S U(2)_{L} \otimes U(1)_{Y}$ Standard Model (SM). The neutrino right handed components $\gamma_{l L}^{o c}$ are not included in the spectrum of the SM which has only one scalar Higgs doublet, implying massless neutrinos at the tree level. This result holds at all orders in perturbation theory and also when nonperturbative effects are taken into account due to the existence of an exact baryon minus lepton number $(B-L)$ symmetry, even if $(B+L)$ is violated by weak sphaleron configurations. Nevertheless, neutrinos oscillate [1-5] which implies that at least two of them have small but nonzero masses.

Masses for neutrinos require physics beyond the SM connected either to the existence of $\nu_{l L}^{0 c}$ and/or to the breaking of the $(B-L)$ symmetry. If right handed neutrinos exist, the Yukawa terms lead, after electroweak symmetry breaking, to Dirac neutrino masses, requiring Yukawa coupling constants for neutrinos $h_{v}^{\phi} \leq 10^{-13}$. But $v_{l L}^{0 c}$, singlets under the SM gauge group, can acquire large Majorana masses and turn on the see-saw mechanism [6-10], an appealing and natural scenery for neutrino mass generation.
Alternatively, the left handed neutrinos, members of the SM lepton doublets $\psi_{l L}=\left(\nu_{l}, l^{-}\right)_{L}^{T}$, can also acquire a Majorana mass $m_{v}$ which carries weak isospin $I=1$ and violates lepton number $L$ by two units, generated via nonrenormalizable operators of the form $\left(\bar{\psi}_{l L}^{c} \widetilde{\phi}^{*}\right)\left(\widetilde{\phi}^{\dagger} \psi_{l^{\prime} L}\right)$, where $\phi=\left(\phi^{+}, \phi^{0}\right)$ is the SM Higgs doublet and $\widetilde{\phi}=i \sigma_{2} \phi^{*}$. This dimension five operator is able to generate type I, type II, and type III see-saw mechanisms by using for heavy fields an $S U(2)_{L}$ singlet fermion, triplet scalar, and triplet fermion, respectively (in this regard, see $[11,12]$ ).

The mechanism of coupling two standard model lepton doublets with a Higgs triplet $\Delta$ which in turn develops nonzero vacuum expectation values (VEV), breaking in this way the lepton number spontaneously, implies in turn the existence of a Majoron [13], particle ruled out experimentally by the $Z$ line shape measurements $[14,15]$ (a singlet Majoron may still survive but with large constraints $[16,17])$.

The variant Zee mechanism [18-20] can be implemented, when the $L=2$ Lorentz scalar $\psi_{l L} C \psi_{l^{\prime} L}$ (with $C$ the charge conjugation matrix) is coupled to an $S U(2)_{L}$ charged singlet $h^{+}$with $L=-2$, introducing next a new scalar doublet $\phi^{\prime}$ and breaking the $L$ symmetry explicitly in the scalar potential with a term of the form $\phi \phi^{\prime} h^{+}$. In this way, neutrino Majorana 
masses are generated by one-loop quantum effects and the unwanted Majoron is not present.

The second Higgs doublet $\phi^{\prime}$ can be avoided by introducing instead a double charged Higgs singlet $k^{++}$which couples to the single charged one by the trilinear coupling $k^{++} h^{-} h^{-}$ and to the right handed charged leptons singlets $l_{R}^{-}$via a term of the form $l_{R}^{-} C l_{R}^{\prime-} k^{++}$, generating in this way Majorana small masses via two-loop quantum effects by what is known as the Zee-Babu mechanism [21, 22].

More examples of generation of neutrino masses via quantum effects can be found in the systematic study presented in [23].

This situation motivates us to perform an extensive analysis of the lepton number symmetry in the most relevant 3-3-1 models. In particular, we are interested in the gauging away mechanism of the Majoron that the so-called economical model presents, due to the subtle connection between the lepton number generator $L$ and one of the $S U(3)_{L}$ generators.

This paper is organized as follows. In Section 2 we review the charge assignment and the gauge boson content of the 3 3-1 models in general, in Section 3 the four possibilities for lepton number violation in the context of the minimal version of the 3-3-1 model are presented, and in Section 4 we classify all the 3-3-1 models without exotic electric charges and repeat the analysis presented in Section 3, but now for the so-called 3-3-1 model with right handed neutrinos. Then in Section 5 we do the general analysis for the 8 different 3-3-1 models without exotic electric charges with 3 families. In Section 6 the so-called economical model is studied and, finally, our conclusions are presented in Section 7.

\section{3-3-1 Models}

Some interesting extensions of the SM are based on the local gauge group $S U(3)_{c} \otimes S U(3)_{L} \otimes U(1)_{x}$ (3-3-1 for short) in which the weak sector of the $S M$ is extended to $S U(3)_{L} \otimes U(1)_{x}$. Several models for this gauge structure have been constructed so far.

For the 3-3-1 models, the most general electric charge operator in the extended electroweak sector is

$$
Q=a \lambda_{3}+\frac{1}{\sqrt{3}} b \lambda_{8}+x I_{3}
$$

where $\lambda_{\alpha}, \alpha=1,2, \ldots, 8$ are the Gell-Mann matrices for $S U(3)_{L}$ normalized as $\operatorname{Tr}\left(\lambda_{\alpha} \lambda_{\beta}\right)=2 \delta_{\alpha \beta}$ and $I_{3}=\operatorname{Dg}(1,1,1)$ is the diagonal $3 \times 3$ unit matrix. $a=1 / 2$ if one assumes that the isospin $S U(2)_{L}$ of the SM is entirely embedded in $S U(3)_{L} ; b$ is a free parameter which defines the different possible models, and the $x$ values are obtained by anomaly cancellation. For $A_{\mu}^{\alpha}$, the 8 gauge fields of $S U(3)_{L}, x=0$ and thus we may write

$$
\sum_{\alpha} \lambda_{\alpha} A_{\mu}^{\alpha}=\sqrt{2}\left(\begin{array}{ccc}
D_{1 \mu}^{0} & W_{\mu}^{+} & K_{\mu}^{(b+1 / 2)} \\
W_{\mu}^{-} & D_{2 \mu}^{0} & K_{\mu}^{(b-1 / 2)} \\
K_{\mu}^{-(b+1 / 2)} & K_{\mu}^{-(b-1 / 2)} & D_{3 \mu}^{0}
\end{array}\right),
$$

where $D_{1 \mu}^{0}=A_{\mu}^{3} / \sqrt{2}+A_{\mu}^{8} / \sqrt{6}, D_{2 \mu}^{0}=-A_{\mu}^{3} / \sqrt{2}+A_{\mu}^{8} / \sqrt{6}$, and $D_{3 \mu}^{0}=-2 A_{\mu}^{8} / \sqrt{6}$. The upper indices on the gauge bosons stand for the electric charge of the particles, some of them being functions of the $b$ parameter.

\section{The Minimal Model}

In $[24,25]$ it has been shown that, for $b=3 / 2$, the following fermion structure is free of all the gauge anomalies: $\psi_{l L}^{T}=$ $\left(v_{l}^{0}, l^{-}, l^{+}\right)_{L} \sim(1,3,0), Q_{i L}^{T}=\left(d_{i}, u_{i}, X_{i}\right)_{L} \sim\left(3,3^{*},-1 / 3\right)$, $Q_{3 L}^{T}=\left(u_{3}, d_{3}, Y\right) \sim(3,3,2 / 3)$, where $l=e, \mu, \tau$ is a family lepton index, $i=1,2$ for the first two quark families, and the numbers after the similarity sign mean 3-3-1 representations. The right handed fields are $u_{a L}^{c} \sim\left(3^{*}, 1,-2 / 3\right), d_{a L}^{c} \sim$ $\left(3^{*}, 1,1 / 3\right), X_{i L}^{c} \sim\left(3^{*}, 1,4 / 3\right)$, and $Y_{L}^{c} \sim\left(3^{*}, 1,-5 / 3\right)$, where $a=1,2,3$ is the quark family index and there are two exotic quarks with electric charge $-4 / 3\left(X_{i}\right)$ and other with electric charge $5 / 3(Y)$. This version is called minimal in the literature, because its lepton content is just the one present in the SM.

For this model, the minimal scalar content required to break the symmetry, giving a realistic mass spectrum, consists of three triplets and one sextet: $\eta^{T}=\left(\eta^{0}, \eta_{1}^{-}, \eta_{2}^{+}\right) \sim(1,3,0)$, $\rho^{T}=\left(\rho^{+}, \rho^{0}, \rho^{++}\right) \sim(1,3,1), \chi^{T}=\left(\chi^{-}, \chi^{--}, \chi^{0}\right) \sim(1,3,-1)$, and

$$
S=\left(\begin{array}{ccc}
\sigma_{1}^{0} & s_{1}^{+} & s_{2}^{-} \\
s_{1}^{+} & s_{1}^{++} & \sigma_{2}^{0} \\
s_{2}^{-} & \sigma_{2}^{0} & s_{2}^{--}
\end{array}\right) \sim\left(1,6^{*}, 0\right)
$$

The scalars have Yukawa couplings to the leptons and quarks as follows:

$$
\begin{aligned}
\mathscr{L}_{1}^{l}= & h_{l l^{\prime}}^{\eta} \eta \psi_{l L} C \psi_{l^{\prime} L}+h_{l l^{\prime}}^{s} \psi_{l L} S C \psi_{l^{\prime} L}+\text { h.c. }, \\
\mathscr{L}_{1}^{q}= & h_{i a}^{u} Q_{i L}^{T} \rho C u_{a L}^{c}+h_{i a}^{d} Q_{i L}^{T} \eta C d_{a L}^{c} \\
& +h_{i j}^{X} Q_{i L}^{T} \chi C X_{j L}^{c}+h_{3 a}^{d} Q_{3 L}^{T} \rho^{*} C d_{a L}^{c} \\
& +h_{3 a}^{u} Q_{3 L}^{T} \eta^{*} C u_{a L}^{c}+h^{Y} Q_{3 L}^{T} \chi^{*} C Y_{L}^{c}+\text { h.c. },
\end{aligned}
$$

with vacuum expectation values (VEV) given by $\left\langle\eta^{0}\right\rangle=v_{1}$, $\left\langle\rho^{0}\right\rangle=v_{2},\left\langle\chi^{0}\right\rangle=v_{3},\left\langle\sigma_{1}^{0}\right\rangle=v_{4}$, and $\left\langle\sigma_{2}^{0}\right\rangle=v_{4}^{\prime}$.

One of the main characteristics of this model is the fact that the lepton number $L$ is not a good quantum number because both, the charged lepton and its antiparticle, are in the same multiplet; as a consequence, $L$ does not commute with the electroweak extended gauge symmetry.

The assignment of $L$ starts with the SM assignments [26]:

$$
\begin{aligned}
& L\left(l_{L}^{-}, v_{l L}\right)=-L\left(l_{L}^{+}\right)=1, \\
& L\left(u_{a L}, u_{a L}^{c}, d_{a L}, d_{a L}^{c}, W_{\mu}^{ \pm}, D_{1 \mu}^{0}, D_{2 \mu}^{0}, D_{3 \mu}^{0}\right)=0 ;
\end{aligned}
$$


then, looking to the Yukawa interactions of the SM particles and imposing $L=0$ in the covariant derivative imply

$$
\begin{aligned}
& L\left(K^{++}, K^{+}, Y_{L}, X_{i L}^{c}\right)=-2 \\
& L\left(K^{--}, K^{-}, X_{i L}, Y_{L}^{c}\right)=2 .
\end{aligned}
$$

For the scalars, $L$ is assigned by inspection of the Yukawa coupling constants and one finds

$$
\begin{aligned}
& L\left(\chi^{-}, \chi^{--}, s_{2}^{--}\right)=2, \\
& L\left(\eta_{2}^{+}, \rho^{++}, \sigma_{1}^{0}, s_{1}^{+}, s_{1}^{++}\right)=-2, \\
& L\left(\eta^{0}, \eta_{1}^{-}, \chi^{0}, \rho^{+}, \rho^{0}, \sigma_{2}^{0}, s_{2}^{-}\right)=0 .
\end{aligned}
$$

Notice that $X_{i}$ and $Y$ are bi-leptoquarks and $K^{+}, K^{-}, K^{++}$, and $K^{--}$are bi-lepton gauge bosons. Finally, the physical gauge bosons related to the neutral currents of the model have $L=$ 0 .

It is interesting to notice that the above lepton numbers of the individual components of each multiplet can be written as [27]:

$$
L=\frac{2 \lambda_{8}}{\sqrt{3}}+\mathscr{L} I_{3},
$$

where $\mathscr{L}$ is a global symmetry of the Lagrangian which is not broken by the VEV and is related to the following assignment: $\mathscr{L}\left(\psi_{l L}\right)=1 / 3, \mathscr{L}\left(Q_{i L}\right)=2 / 3, \mathscr{L}\left(Q_{3 L}, \eta, S, \rho\right)=-2 / 3, \mathscr{L}(\chi)=$ $4 / 3, \mathscr{L}\left(X_{i L}^{c}\right)=-2, \mathscr{L}\left(Y_{L}^{c}\right)=2$, and $\mathscr{L}\left(u_{a}^{c}, d_{a}^{c}, A_{\alpha \mu}\right)=0$.

The former analysis shows that since $L\left(\eta^{0}, \chi^{0}, \rho^{0}, \sigma_{2}^{0}\right)=$ 0 , the only place where the $L$ number can be spontaneously violated is in $\sigma_{1}^{0}$, but it may be explicitly violated in the scalar potential. As a matter of fact, a term like

$$
\begin{aligned}
V_{L V}= & f_{1} \eta S \eta+f_{2} S S S+\kappa_{1}\left(\chi^{\dagger} \eta\right)\left(\rho^{\dagger} \eta\right) \\
& +\kappa_{2} \eta^{\dagger} S \chi \rho+\kappa_{3} \chi \rho S S+\text { h.c. }
\end{aligned}
$$

explicitly violates $\Delta \mathscr{L}=\Delta L= \pm 2$ when all the VEV are zero, leaving $\lambda_{8}$ unbroken. Then, the four possibilities of lepton number violation in the context of this model are thus as follows.

(1) $V_{L V}=0$ and $\langle S\rangle=0$. This is the minimal 3-3-1 PisanoPleitez-Frampton model where total lepton number is conserved and neutrinos are massless particles. Consequently, this version of the model is in conflict with the existence of massive neutrinos.

(2) $V_{L V}=0$ but $\left\langle\sigma_{1}^{0}\right\rangle \neq 0$. In this case the lepton number is spontaneously broken leading to a triplet Majoron. This case has been analyzed in [28, 29].

(3) $V_{L V} \neq 0$ and $\left\langle\sigma_{1}^{0}\right\rangle=0$. $L$ is violated explicitly and nonzero masses for neutrinos can be generated from quantum corrections.

(4) The case for $V_{L V} \neq 0$ and $\left\langle\sigma_{1}^{0}\right\rangle \neq 0$ is also possible, with a rich phenomenology which may include a light pseudo Goldstone Majoron [30].
TABLE 1: Anomalies for 3-3-1 fermion fields structures.

\begin{tabular}{lcccccc}
\hline Anomalies & $S_{1}$ & $S_{2}$ & $S_{3}$ & $S_{4}$ & $S_{5}$ & $S_{6}$ \\
\hline$\left[S U(3)_{C}\right]^{2} U(1)_{X}$ & 0 & 0 & 0 & 0 & 0 & 0 \\
{$\left[S U(3)_{L}\right]^{2} U(1)_{X}$} & $-2 / 3$ & $-1 / 3$ & 1 & 0 & 0 & -1 \\
{$[G r a v]^{2} U(1)_{X}$} & 0 & 0 & 0 & 0 & 0 & 0 \\
{$\left[U(1)_{X}\right]^{3}$} & $10 / 9$ & $8 / 9$ & $-12 / 9$ & $-6 / 9$ & $6 / 9$ & $12 / 9$ \\
{$\left[S U(3)_{L}\right]^{3}$} & 1 & -1 & -3 & 3 & -3 & 3 \\
\hline
\end{tabular}

\section{3-3-1 Models without Exotic Electric Charges}

If one wishes to avoid exotic electric charges as the ones present in the minimal model, one must choose $b=1 / 2$, in (1). Following $[31,32]$ we can find six sets of fermions which contain the antiparticles of the charged particles which are

(i) $S_{1}=\left[\left(\nu_{\alpha}^{0}, \alpha^{-}, E_{\alpha}^{-}\right) ; \alpha^{+} ; E_{\alpha}^{+}\right]_{L}$ with quantum numbers $(1,3,-2 / 3) ;(1,1,1)$; and $(1,1,1)$, respectively,

(ii) $S_{2}=\left[\left(\alpha^{-}, v_{\alpha}, N_{\alpha}^{0}\right) ; \alpha^{+}\right]_{L}$ with quantum numbers $\left(1,3^{*}\right.$, $-1 / 3)$ and $(1,1,1)$, respectively,

(iii) $S_{3}=\left[(d, u, U) ; u^{c} ; d^{c} ; U^{c}\right]_{L}$ with quantum numbers $(3$, $\left.3^{*}, 1 / 3\right) ;\left(3^{*}, 1,-2 / 3\right) ;\left(3^{*}, 1,1 / 3\right)$; and $\left(3^{*}, 1,-2 / 3\right)$, respectively,

(iv) $S_{4}=\left[(u, d, D) ; u^{c} ; d^{c} ; D^{c}\right]_{L}$ with quantum numbers $(3,3,0) ;\left(3^{*}, 1,-2 / 3\right) ;\left(3^{*}, 1,1 / 3\right)$; and $\left(3^{*}, 1,1 / 3\right)$, respectively,

(v) $S_{5}=\left[\left(e^{-}, v_{e}, N_{1}^{0}\right) ;\left(E^{-}, N_{2}^{0}, N_{3}^{0}\right) ;\left(N_{4}^{0}, E^{+}, e^{+}\right)\right]_{L}$ with quantum numbers $\left(1,3^{*},-1 / 3\right) ;\left(1,3^{*},-1 / 3\right)$; and $\left(1,3^{*}, 2 / 3\right)$, respectively,

(vi) $S_{6}=\left[\left(\nu_{e}, e^{-}, E_{1}^{-}\right) ;\left(E_{2}^{+}, N_{1}^{0}, N_{2}^{0}\right) ;\left(N_{3}^{0}, E_{2}^{-}, E_{3}^{-}\right) ; e^{+} ; E_{1}^{+}\right.$; $\left.E_{3}^{+}\right]_{L}$ with quantum numbers $(1,3,-2 / 3) ;(1,3,1 / 3)$; $(1,3,-2 / 3) ;(111),(111)$; and (111), respectively. Table 1

The different anomalies for these six sets are [31] found in

With this table, anomaly-free models, without exotic electric charges, can be constructed for one, two, or more families.

As noted in [31], there are eight three-family models that are anomaly free, which are:

(i) Model A: with right handed neutrinos $3 S_{2}+S_{3}+2 S_{4}$,

(ii) Model B: with exotic electrons $3 S_{1}+2 S_{3}+S_{4}$,

(iii) Model C: with unique lepton generation one (three different lepton families) $S_{1}+S_{2}+S_{3}+2 S_{4}+S_{5}$,

(iv) Model D: with unique lepton generation two $S_{1}+S_{2}+$ $2 S_{3}+S_{4}+S_{6}$,

(v) Model E: hybrid one (two different lepton structures) $S_{3}+2 S_{4}+2 S_{5}+S_{6}$,

(vi) Model F: hybrid two $2 S_{3}+S_{4}+S_{5}+2 S_{6}$,

(vii) Model G: carbon copy one (three identical families as in the SM) $3\left(S_{4}+S_{5}\right)$,

(viii) Model H: carbon copy two $3\left(S_{3}+S_{6}\right)$. 
4.1. The 3-3-1 Model with Right Handed Neutrinos. Introduced in $[33,34]$, it has the following 3-3-1 anomaly-free fermion structure:

$$
\begin{aligned}
& \psi_{l L}^{T}=\left(l^{-}, v_{l}^{0}, N_{l}^{0}\right)_{L} \sim\left(1,3^{*},-\frac{1}{3}\right), \quad l_{L}^{+} \sim(1,1,1), \\
& Q_{i L}^{T}=\left(u_{i}, d_{i}, D_{i}\right)_{L} \sim(3,3,0), \\
& Q_{3 L}^{T}=\left(d_{3}, u_{3}, U\right)_{L} \sim\left(3,3^{*}, \frac{1}{3}\right),
\end{aligned}
$$

where $l=e, \mu, \tau$ is a family lepton index, $N_{l L}^{0}$ stands for electrically neutral Weyl state, and $i=1,2$ for the first two quark families. The right handed quark fields are

$$
\begin{array}{ll}
u_{a L}^{c} \sim\left(3^{*}, 1,-\frac{2}{3}\right), & d_{a L}^{c} \sim\left(3^{*}, 1, \frac{1}{3}\right), \\
D_{i L}^{c} \sim\left(3^{*}, 1, \frac{1}{3}\right), & U_{L}^{c} \sim\left(3^{*}, 1,-\frac{2}{3}\right),
\end{array}
$$

where again $a=1,2,3$ is the quark family index and there are two exotic quarks with electric charge $-1 / 3\left(D_{i}\right)$ and other with electric charge $2 / 3(U)$.

The minimal scalar content required to break the symmetry, giving a realistic mass spectrum, consists now of only three triplets $[33,34]$ :

$$
\begin{aligned}
& \rho^{T}=\left(\rho_{1}^{0}, \rho_{2}^{+}, \rho_{3}^{+}\right) \sim\left(1,3^{*}, \frac{2}{3}\right), \\
& \eta^{T}=\left(\eta_{1}^{-}, \eta_{2}^{0}, \eta_{3}^{0}\right) \sim\left(1,3^{*},-\frac{1}{3}\right), \\
& \chi^{T}=\left(\chi_{1}^{-}, \chi_{2}^{0}, \chi_{3}^{0}\right) \sim\left(1,3^{*},-\frac{1}{3}\right),
\end{aligned}
$$

with VEV given by $\left\langle\rho^{0}\right\rangle^{T}=\left(v_{1}, 0,0\right),\left\langle\eta^{0}\right\rangle^{T}=\left(0, v_{2}, 0\right)$, and $\left\langle\chi^{0}\right\rangle^{T}=(0,0, V)$.

A careful analysis of the Yukawa terms for the lepton sector

$$
\mathscr{L}_{\text {lep }}^{Y}=h_{l l^{\prime}}^{e} \rho^{*} \psi_{l L} C l_{L}^{+\prime}+h_{l l^{\prime}} \rho \psi_{l L} C \psi_{l^{\prime} L},
$$

shows that $N_{l L}^{0}$, the third component of the fermion triplet $\left(1,3^{*},-1 / 3\right)$, must be identified with $\nu_{l L}^{0 c}$, the antiparticle of $\nu_{l L}^{0}$. As a consequence, $L$ is not a good quantum number in the context of this model, because $L$ does not commute with the symmetry $S U(3)_{L} \otimes U(1)_{X}$.

Doing a similar analysis to the one presented for the minimal model, we obtain the following lepton number assignments [35]:

$$
\begin{aligned}
& L\left(l_{L}^{-}, v_{l L}^{0}\right)=-L\left(l_{L}^{+}, v_{l L}^{0 c}\right)=1, \\
& L\left(u_{a L}, u_{a L}^{c}, d_{a L}, d_{a L}^{c}, W_{\mu}^{ \pm}, D_{1 \mu}^{0}, D_{2 \mu}^{0}, D_{3 \mu}^{0}\right)=0 ; \\
& L\left(K^{+}, K^{0}, U_{L}, D_{i L}^{c}\right)=-L\left(K^{-}, \bar{K}^{0}, D_{i L}, U_{L}^{c}\right)=-2, \\
& L\left(\chi_{1}^{-}, \chi_{2}^{0}\right)=-L\left(\rho_{3}^{+}, \eta_{3}^{0}\right)=2, \\
& L\left(\rho_{1}^{0}, \rho_{2}^{+}, \eta_{1}^{-}, \eta_{2}^{0}, \chi_{3}^{0}\right)=0 .
\end{aligned}
$$

Notice that the new quarks $D_{i}$ and $U$ are bileptoquarks and $K^{+}, K^{-}, K^{0}$, and $\bar{K}^{0}$ are bi-lepton gauge bosons.

Again, (8) can be used to write the previous lepton number assignment using now the following $\mathscr{L}$ values: $\mathscr{L}\left(\psi_{l L}\right)=1 / 3, \mathscr{L}\left(Q_{i L}\right)=2 / 3, \mathscr{L}\left(Q_{3 L}, \eta, \rho\right)=-2 / 3$, $\mathscr{L}(\chi)=4 / 3, \mathscr{L}\left(D_{i L}^{c}\right)=-2, \mathscr{L}\left(U_{L}^{c}\right)=2, \mathscr{L}\left(l_{L}^{+}\right)=-1$, and $\mathscr{L}\left(u_{a L}^{c}, d_{a L}^{c}, A_{\alpha \mu}\right)=0$, values in agreement with the one presented in [35].

For this model, the quark mass spectrum was analyzed in [35], using only the following lepton number conservation Yukawa potential:

$$
\begin{aligned}
\mathscr{L}_{\mathrm{LNC}}^{Y}= & h^{U} \chi^{*} Q_{3 L} C U_{L}^{c}+h_{i j}^{D} \chi Q_{i L} C D_{j L}^{c} \\
& +h_{a}^{d} \rho^{*} Q_{3 L} C d_{a L}^{c}+h_{i a}^{u} \rho Q_{i L} C u_{a L}^{c} \\
& +h_{3 a} \eta^{*} Q_{3 L} C u_{a L}^{c}+h_{i a} \eta Q_{i L} C d_{a L}^{c}+\text { h.c. }
\end{aligned}
$$

which conserves both the global numbers $L$ and $\mathscr{L}$. But the most general Yukawa potential for quarks must also include the following terms:

$$
\begin{aligned}
\mathscr{L}_{\mathrm{LNV}}^{Y}= & h_{a}^{u} \chi^{*} Q_{3 L} C u_{a L}^{c}+h_{i a}^{d} \chi Q_{i L} C d_{a L}^{c} \\
& +h_{i}^{D} \rho^{*} Q_{3 L} C D_{i L}^{c}+h_{i}^{U} \rho Q_{i L} C U_{L}^{c} \\
& +h^{U} \eta^{*} Q_{3 L} C U_{L}^{c}+h_{i j}^{D} \eta Q_{i L} C D_{j L}^{c}+\text { h.c. },
\end{aligned}
$$

which explicitly violates the global numbers $L$ and $\mathscr{L}$. This avoids the possible existence of a Majoron in the context of this model.

The Yukawa Lagrangian for the neutral leptons extracted from (13), and in the basis $\left(\nu_{1}, v_{2}, v_{3}, v_{1}^{c}, v_{2}^{c}, v_{3}^{c}\right)$, produces the following tree level neutrino mass matrix:

$$
M=\left(\begin{array}{cccccc}
0 & 0 & 0 & 0 & a & b \\
0 & 0 & 0 & -a & 0 & c \\
0 & 0 & 0 & -b & -c & 0 \\
0 & -a & -b & 0 & 0 & 0 \\
a & 0 & -c & 0 & 0 & 0 \\
b & c & 0 & 0 & 0 & 0
\end{array}\right),
$$

where the entries are Dirac masses at the SM scale, times Yukawa couplings, with eigenvalues $\left(0,0, \pm m_{v}, \pm m_{v}\right)$ where $m_{v}=\sqrt{a^{2}+b^{2}+c^{2}}$ which stands for three Dirac neutrinos, one massless and two degenerated. The model is viable only for very small Yukawa couplings constants and radiative corrections able to remove the degeneracies (analysis done to a limited extent in [35]).

In general $\chi_{2}^{0}$ and $\eta_{3}^{0}$ can have a VEV different from zero which could imply spontaneous symmetry breaking of the lepton number $L$. But $L$ can also be broken explicitly in the scalar potential by terms of the form

$$
\begin{aligned}
V_{L V}^{\prime}= & \mu \chi^{\dagger} \eta+\eta^{\dagger} \chi\left(\kappa_{1}|\rho|^{2}+\kappa_{2}|\eta|^{2}+\kappa_{3}|\chi|^{2}\right) \\
& +\kappa_{4}\left|\chi^{\dagger} \eta\right|^{2}+\kappa_{5}\left(\eta^{\dagger} \rho\right)\left(\rho^{\dagger} \chi\right)+\text { h.c., }
\end{aligned}
$$


which again satisfy $\Delta \mathscr{L}=\Delta L= \pm 2$ when all the VEV are zero, leaving $\lambda_{8}$ to be broken explicitly.

As in the minimal model there are four different cases.

(1) $V_{V L}^{\prime}=0,\left\langle\chi_{2}^{0}\right\rangle=\left\langle\eta_{3}^{0}\right\rangle=0$. The total lepton number is conserved and the neutrinos can pick up only Dirac type masses.

(2) $V_{V L}^{\prime}=0,\left\langle\chi_{2}^{0}\right\rangle \neq 0$ and $/$ or $\left\langle\eta_{3}^{0}\right\rangle \neq 0$. The lepton number $L$ is now spontaneously violated. This case has been analyzed in [36] where a CP odd Majoron was found.

(3) $V_{V L}^{\prime} \neq 0,\left\langle\chi_{2}^{0}\right\rangle=\left\langle\eta_{3}^{0}\right\rangle=0 . L$ is explicitly violated and again nonzero masses for neutrinos can be generated by quantum effects.

(4) Again, $V_{V L}^{\prime} \neq 0,\left\langle\chi_{2}^{0}\right\rangle \neq 0$, and/or $\left\langle\eta_{3}^{0}\right\rangle \neq 0$ is also possible, leading to a phenomenology with the presence of a light pseudo Goldstone Majoron.

\section{The Neutral Sector}

To present the kind of analysis we are aimed to, let us concentrate on Model D to start with.

The lepton fields for this particular model are included in the structure $S_{1}+S_{2}+S_{6}$ which contains 21 two component spinors, including seven neutral Weyl states. Let us write them in the following way:

$$
\begin{aligned}
& \psi_{1 L}=\left(v_{1}, l_{1}^{-}, E_{0}^{-}\right)_{L} \sim\left(1,3,-\frac{2}{3}\right), \\
& l_{1 L}^{+} \sim(1,1,1), \quad E_{0 L}^{+} \sim(1,1,1) \\
& \psi_{2 L}=\left(l_{2}^{-}, v_{2}, N_{0}^{0}\right)_{L} \sim\left(1,3^{*},-\frac{1}{3}\right), \quad l_{2 L}^{+} \sim(1,1,1), \\
& \psi_{3 L}=\left(v_{3}, l_{3}^{-}, E_{1}^{-}\right)_{L} \sim\left(1,3,-\frac{2}{3}\right), \\
& l_{3 L}^{+} \sim(1,1,1), \quad E_{1 L}^{+} \sim(1,1,1) \\
& \psi_{4 L}=\left(E_{2}^{+}, N_{1}^{0}, N_{2}^{0}\right)_{L} \sim\left(1,3, \frac{1}{3}\right), \\
& \psi_{5 L}=\left(N_{3}^{0}, E_{2}^{-}, E_{3}^{-}\right)_{L} \sim\left(1,3,-\frac{2}{3}\right), \quad E_{3 L}^{+} \sim(1,1,1),
\end{aligned}
$$

with the 3-3-1 quantum numbers given in parenthesis.

Using the scalars of (12) with the VEV as stated, the mass matrix for the neutral sector in the basis $\left(\nu_{1}, \nu_{2}, v_{3}, N_{0}^{0}, N_{1}^{0}, N_{2}^{0}, N_{3}^{0}\right)$ is now of the form

$$
M_{n}=\left(\begin{array}{ccccccc}
0 & 0 & 0 & 0 & A & -a & 0 \\
0 & 0 & 0 & 0 & M & 0 & 0 \\
0 & 0 & 0 & 0 & B & -b & 0 \\
0 & 0 & 0 & 0 & 0 & M & 0 \\
A & M & B & 0 & 0 & 0 & G \\
-a & 0 & -b & M & 0 & 0 & -d \\
0 & 0 & 0 & 0 & G & -d & 0
\end{array}\right),
$$

TABLE 2: Tree level neutrinos sectors.

\begin{tabular}{lccc}
\hline Model & $\begin{array}{c}\text { Number of Weyl } \\
\text { neutral states }\end{array}$ & $\begin{array}{c}\text { Massless } \\
\text { Weyl states }\end{array}$ & $\begin{array}{c}\text { Dirac states at } \\
\text { the EW scale }\end{array}$ \\
\hline A & 6 & 2 & 2 \\
B & 3 & 3 & 0 \\
C & 8 & 0 & 3 \\
D & 7 & 3 & 0 \\
E & 14 & 0 & 3 \\
F & 13 & 0 & 1 \\
G & 12 & 0 & 3 \\
H & 15 & 0 & 4 \\
\hline
\end{tabular}

where the $M$ value is related to a GUT mass scale coming from the bare mass term $\psi_{2 L} C \psi_{4 L}+$ h.c; $A, B$, and $C$ are mass terms at the TeV scale $V$, and $a, b$, and $c$ are mass terms at the electroweak scale $v \sim v_{1} \sim v_{2}$. The diagonalization of the former mass matrix produces two Dirac massive spinors with masses at the GUT scale and three Weyl massless states that we can associate with the detected solar and atmospheric oscillating neutrinos.

So, up to this point the model has the potential to be consistent with the neutrino phenomenology. But the question is if the three Weyl states remain massless or if they may pick up small radiative masses in the context of the model, or a simple extension of it, something out of the reach of the analysis presented here.

5.1. General Analysis for 3 Families. Analysis similar to the previous one has been carried through for the neutral fermion sector of the eight anomaly-free lepton structures enumerated in Section 4. The results are presented in Table 2.

According to this table, only models B and D fulfill the natural condition of having 3 tree-level zero mass neutrinos, which may pick up nonzero masses via radiative corrections, with or without the addition of new ingredients. Some other structures may become realistic if new fields are added, and/or if some Yukawa coupling constants are fine tuned to very small values, and/or if discreet symmetries which forbids Yukawa terms are imposed, and so forth.

Let us see this in the following example.

5.2. The 3-3-1 Model with Exotic Electrons. To see what kind of new ingredients are needed in order to provide masses to the neutral fields in these 3-3-1 models without exotic electric charges, let us briefly view the situation for model B which was introduced in the literature for the first time in [37]. The neutral fermion sector for this model has been studied in some detail in [38,39], but the approach here is simpler.

The anomaly-free fermion structure for this model is [37]:

$$
\begin{aligned}
& \psi_{l L}^{T}=\left(\nu_{l}^{0}, l^{-}, E_{l}^{-}\right)_{L} \sim\left(1,3,-\frac{2}{3}\right), \\
& l_{L}^{+} \sim(1,1,1), \quad E_{l L}^{+} \sim(1,1,1), \\
& Q_{i L}^{T}=\left(d_{i}, u_{i}, U_{i}\right)_{L} \sim\left(3,3^{*}, \frac{1}{3}\right),
\end{aligned}
$$




$$
\begin{aligned}
& Q_{3 L}^{T}=\left(u_{3}, d_{3}, D\right) \sim(3,3,0), \\
& u_{a L}^{c} \sim\left(3^{*}, 1,-\frac{2}{3}\right), \quad U_{i L}^{c} \sim\left(3^{*}, 1,-\frac{2}{3}\right), \\
& d_{a L}^{c} \sim\left(3^{*}, 1, \frac{1}{3}\right), \quad D_{L}^{c} \sim\left(3^{*}, 1, \frac{1}{3}\right),
\end{aligned}
$$

where $l=e, \mu, \tau$ is a lepton family index, $E_{l}^{-}$stands for three exotic electron fields, $i=1,2$ for the first two quark families, $a=1,2,3$ is again the quark family index, and there are two exotic quarks with electric charge $2 / 3\left(U_{i}\right)$ and other one with electric charge $-1 / 3(D)$. This model does not contain right handed neutrino fields.

The gauge boson and scalar sectors for this model are exactly the same ones to that for the model with right handed neutrinos [33]; but the big differences are that now the lepton number $L$ is a good quantum number of the model and the gauge bosons do not carry lepton number at all, neither the exotic quarks. The scalars $(\eta, \rho, \chi)$ introduced have also $L=0$, the lepton number cannot be broken spontaneously, and, as a consequence, the neutrinos remain massless even with the inclusion of the radiative corrections.

In what follows and in order to simplify matters and make this model more predictable, we consider only the set of two scalar triplets $\chi$ and $\rho$ instead of the set of three triplets proposed in the original paper [37], or the much more complex structure introduced in [38]. Also, let us take the VEV to be $\langle\chi\rangle^{T}=(0, v, V)$ and $\langle\rho\rangle^{T}=\left(v_{1}, 0,0\right)$ (as in the Economical 3-3-1 model [40] which is going to be studied next). The Yukawa couplings of the leptons to this scalars are now

$$
\mathscr{L}_{2}^{l}=\sum_{l, l^{\prime}}\left[\left(\psi_{l L}^{T} \cdot \chi\right) C\left(h_{l l^{\prime}}^{e} l_{L}^{\prime+}+h_{l l^{\prime}}^{E} E_{l^{\prime} L}^{+}\right)\right]+\text {h.c. }
$$

which for $l, l^{\prime}=e, \mu, \tau$ saturates all the entries of the $6 \times 6$ charged lepton mass matrix and allows tree-level masses only for charged leptons, even though there are in (22) external legs with neutrino fields of the form $\nu_{l L}^{0} \chi_{1}^{-} C\left(h_{l l^{\prime}}^{e} l_{L}^{\prime+}+h_{l l^{\prime}}^{E} E_{l^{\prime} L}^{+}\right)+$ h.c. The possible inclusion of the scalar $\eta$ does not change this situation at all.

Masses for neutrinos can be obtained only by enlarging the model. For this purpose one can introduce a new scalar triplet $\phi=\left(\phi_{1}^{++}, \phi_{2}^{+}, \phi_{3}^{+}\right) \sim(1,3,4 / 3)$ which couples to the spin $1 / 2$ leptons via a term in the Lagrangian of the form

$$
\begin{gathered}
\mathscr{L}_{3}^{l}=\epsilon_{n m p} \sum_{l, l^{\prime}} h_{l l^{\prime}}^{v} \phi^{n} \psi_{l L}^{m} C \psi_{l^{\prime} L}^{p}+h . c ., \\
=\sum_{l l^{\prime}} h_{l l^{\prime}}^{v}\left[\phi_{1}^{++}\left(l_{L}^{-} E_{l^{\prime} L}^{-}-l_{L}^{\prime-} E_{l L}^{-}\right)\right. \\
\left.\quad+\phi_{2}^{+}\left(E_{l L}^{-} \nu_{l^{\prime} L}-E_{l^{\prime} L}^{-} \nu_{l L}\right)+\phi_{3}^{+}\left(v_{l L} l_{L}^{\prime-}-v_{l^{\prime} L} l_{L}^{-}\right)\right] \\
+ \text {h.c. }
\end{gathered}
$$

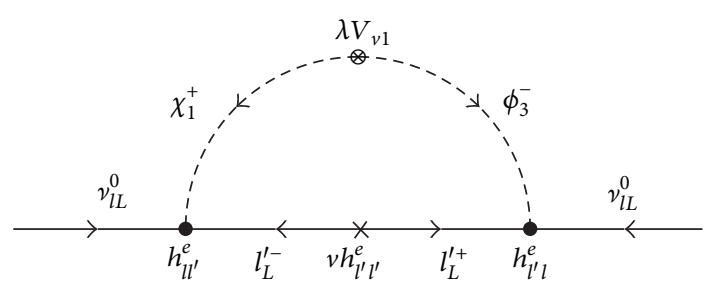

FIGURE 1: Generation of the neutrino masses via the one loop radiative mechanism in the 3-3-1 model with exotic electrons.

which implies lepton number values $L\left(\phi_{1}^{++}, \phi_{2}^{+}, \phi_{3}^{+}\right)=-2$ in order to have it conserved in $\mathscr{L}_{3}^{l}$. Notice that the expression above also provides several external legs with neutrino fields which can be used to generate masses to the neutral fermions via quantum effects.

Since $\langle\phi\rangle=(0,0,0)$, the new scalar fields are not able to break spontaneously the lepton number. But the point is that the lepton symmetry is now explicitly broken in the Lagrangian by a term in the scalar potential of the form $\lambda(\phi \cdot \chi)\left(\rho^{*} \cdot \chi\right)$ which violates lepton number by two units and turns on the Zee radiative mechanism in the context of this 3-3-1 model with exotic electrons. As a matter of fact, all the previous ingredients allow us to draw the diagram in Figure 1 in the context of the field structure presented so far.

Although the scalar sector has three independent fields $(\chi, \rho, \phi)$, its VEV structure is simpler than the one proposed in the original paper [37].

Neutrino masses in the context of the model analyzed in this section were studied for the first time in [41]. The main difference between that paper and this one is that in [41], and in order to implement the Zee-Babu mechanism $[18,19,21]$ for generating neutrino mass terms, a double charged Higgs scalar $S U(3)_{L}$ singlet $k^{++} \sim(1,1,2)$ was used instead of our $\phi$ scalar triplet, which is the new and main ingredient of our analysis. So, both papers address the same problem from two different points of view.

\section{The Economical 3-3-1 Model}

The model was introduced for the first time $[40,42]$ and the quark and lepton content corresponds to the 3-3-1 model with right handed neutrinos presented above; but the scalar sector is modified, becoming minimal in the sense that only two scalar triplets (with a modified VEV structure) are used in order to break the symmetry. They are

$$
\begin{aligned}
& \rho^{T}=\left(\rho_{1}^{0}, \rho_{2}^{+}, \rho_{3}^{+}\right) \sim\left(1,3^{*}, \frac{2}{3}\right), \\
& \chi^{T}=\left(\chi_{1}^{-}, \chi_{2}^{0}, \chi_{3}^{0}\right) \sim\left(1,3^{*},-\frac{1}{3}\right),
\end{aligned}
$$

with the VEV given now by $\langle\rho\rangle^{T}=\left(v_{1}, 0,0\right)$ and $\langle\chi\rangle^{T}=$ $(0, v, V)$.

The lepton number $L$ and the global symmetry $\mathscr{L}$ are as given for the model with right handed neutrinos, and (8) and the lepton number assignment in (14) still hold. 
This model has been the subject of several recent studies [42-44] and it has the peculiarity that the lepton number $L$ is spontaneously broken due to the fact that $L\left(\chi_{2}^{0}\right)=2$.

Since the scalar sector is very simple now, the model is highly predictable. As a matter of fact, the full scalar potential consists only of the following six terms [40]:

$$
\begin{aligned}
V(\chi, \rho)= & \mu_{1}^{2}|\chi|^{2}+\mu_{2}^{2}|\rho|^{2}+\kappa_{1}\left|\chi^{\dagger} \chi\right|^{2}+\kappa_{2}\left|\rho^{\dagger} \rho\right|^{2} \\
& +\kappa_{3}|\chi|^{2}|\rho|^{2}+\kappa_{4}\left|\chi^{\dagger} \rho\right|^{2}+\text { h.c. } .
\end{aligned}
$$

A simple calculation shows that both $\mathscr{L}$ and the lepton number $L$ are conserved by $V(\chi, \rho)$ and also by the full Lagrangian, except for some of the following Yukawa interactions which induce masses for the fermions

$$
\begin{aligned}
\mathscr{L}^{Y}= & \mathscr{L}_{\mathrm{LNC}}^{Y}+\mathscr{L}_{\mathrm{LNV}}^{Y} \\
\mathscr{L}_{\mathrm{LNC}}^{Y}= & h^{U} \chi^{*} Q_{3 L} C U_{L}^{c}+h_{i j}^{D} \chi Q_{i L} C D_{j L}^{c} \\
& +h_{a}^{d} \rho^{*} Q_{3 L} C d_{a L}^{c}+h_{i a}^{u} \rho Q_{i L} C u_{a L}^{c} \\
& +h_{l l^{\prime}}^{e} \rho^{*} \psi_{l L} C l_{L}^{\prime+}+h_{l l^{\prime}} \rho \psi_{l L} C \psi_{l^{\prime} L}+\text { h.c. } \\
\mathscr{L}_{\mathrm{LNV}}^{Y}= & h_{a}^{u} \chi^{*} Q_{3 L} C u_{a L}^{c}+h_{i a}^{d} \chi Q_{i L} C d_{a L}^{c} \\
& +h_{i}^{D} \rho^{*} Q_{3 L} C D_{i L}^{c}+h_{i}^{U} \rho Q_{i L} C U_{L}^{c}+\text { h.c. },
\end{aligned}
$$

where the subscripts LNC and LNV indicate lepton number conserving and lepton number violating term, respectively. As a matter of fact, $\mathscr{L}_{\mathrm{LNV}}^{Y}$ violates explicitly $\mathscr{L}$ and $L$ by two units.

After spontaneous breaking of the gauge symmetry the scalar potential develops the following lepton number violating terms:

$$
\begin{aligned}
V_{\mathrm{LNV}}= & v\left[\sqrt{2} H_{\chi}\left(\kappa_{1}|\chi|^{2}+\kappa_{3}|\rho|^{2}\right)\right] \\
& +v \kappa_{4}\left[\rho_{1}^{-}\left(\chi^{\dagger} \rho\right)+\rho_{1}^{+}\left(\rho^{\dagger} \chi\right)\right],
\end{aligned}
$$

where we have defined as usual $\chi_{2}^{0}=v+\left(H_{\chi}+i A_{\chi}\right) / \sqrt{2}$. $H_{\chi}$ and $A_{\chi}$ are the so-called CP even and CP odd (scalar and pseudo scalar) components, and for simplicity we are taking real VEV (CP violation through the scalar exchange has not been considered here).

Notice that the lepton number violating part in (28) is trilinear in the scalar fields, and as expected, $V_{\mathrm{LNV}}=0$ for $v=0$. From the former expression we can identify $A_{\chi}$ as the only candidate for a Majoron in this model.

The minimization of the scalar potential has been done in full detail in [40] (reproduced also in the second paper in [42]). For that purpose two more definitions were introduced: $\rho_{1}^{0}=v_{1}+\left(H_{\rho}+i A_{\rho}\right) / \sqrt{2}$ and $\chi_{3}^{0}=V+\left(H_{\chi}^{\prime}+i A_{\chi}^{\prime}\right) / \sqrt{2}$. An outline of the main results in [40], important for our present discussion, are as follows.

(i) The three CP odd pseudo scalars $A_{\chi}, A_{\chi}^{\prime}$, and $A_{\rho}$, the would be Goldstone bosons, are eaten up by $Z, Z^{\prime}$, and $\left(K^{0}+\bar{K}^{0}\right) / \sqrt{2}$, the real part of the neutral bi-lepton gauge boson. (ii) Out of the three CP even scalars, $\left(v H_{\chi}^{\prime}-V H_{\chi}\right) /$ $\sqrt{v^{2}+V^{2}}$ becomes a would be Goldstone boson eaten up by $i\left(K^{0}-\bar{K}^{0}\right) / \sqrt{2}$, the imaginary part of the neutral bi-lepton gauge boson which picks up $L=2$ via $H_{\chi}$. The other two CP even scalars become the SM Higgs boson and one extra Higgs boson with a heavy mass of order $V$, respectively.

(iii) In the charged scalar sector $\left(\rho_{2}^{ \pm}, \chi_{1}^{ \pm}, \rho_{3}^{ \pm}\right)$there are four would be Goldstone bosons, two of them are $\left(V \chi_{2}^{ \pm}-\right.$ $\left.v_{1} \rho_{3}^{ \pm}\right) / \sqrt{V^{2}+v_{1}^{2}}$ with $L= \pm 2$, eaten up by $K^{ \pm}$, and other two with $L=0$ eaten up by $W^{ \pm}$.

(iv) Two charged scalars remain as physical states.

Counting degrees of freedom tell us that there are in $\chi$ and $\rho$ twelve ones, namely, three CP even, three CP odd, and six charged ones. Eight of them are eaten up by the eight gauge bosons $W^{ \pm}, K^{ \pm}, K^{0}, \bar{K}^{0}, Z$, and $Z^{\prime}$. Four scalars remain as physical states, one of them being the SM Higgs scalar.

Since $L$ is explicitly broken in the context of this model, the most outstanding result in our analysis is that the would be pseudo Goldstone Majoron $A_{\chi}$, the only CP odd electrically neutral scalar with $L=2$, has been eaten up by $\left(K^{0}+\bar{K}^{0}\right) / \sqrt{2}$, the real part of the bi-lepton gauge boson. A clever way to avoid an unwanted Majoron!

A variant of this model was considered in [45] where the fermion mass spectrum was studied with the inclusion of a $Z_{2}$ discrete symmetry which excludes the LNV interactions in the Yukawa potential in (27). For this variant of the model, $\mathscr{L}$ is conserved through the entire Lagrangian; the lepton number $L$ is only spontaneous violated by $V_{\mathrm{LNV}}$ in (28) and the would be Majoron $A_{\chi}$ is gauge away, eaten up by $\left(K^{0}+\right.$ $\left.\bar{K}^{0}\right) / \sqrt{2}$. Notice that being $\mathscr{L}$ a good quantum number, the spontaneous violation of $S U(3)_{L}$ implies the spontaneous violation of $L$ via (8), something that it is now allowed because the fermion sector for $L$ is vector like and thus nonanomalous.

The economical scalar structure presented here is not able to reproduce a consistent quark mass spectrum at tree level. By fortune, a careful analysis combining the renormalizable Yukawa interactions in (26) and (27) and the effective dimension-five operators

$$
\begin{aligned}
\mathscr{L}_{\mathrm{NR}}=\frac{\epsilon_{n m p}}{\Lambda}[ & \chi^{n} \rho^{m} Q_{3 L}^{p} C\left(\lambda_{3}^{U} U_{L}^{c}+\sum_{a=1}^{3} \lambda_{a}^{u} u_{a L}^{c}\right) \\
& \left.+\chi^{* n} \rho^{* m} \sum_{i=1}^{2} Q_{i L}^{p} C\left(\lambda_{i}^{d} D_{L}^{c}+\sum_{a=1}^{3} \lambda_{i a}^{d} d_{a L}^{c}\right)\right]
\end{aligned}
$$

are able to remove the zero quark masses. But the implementation of $\mathscr{L}_{\mathrm{NR}}$ in the former expression requires the introduction of new and heavy scalar fields.

But there remains the question of the quantum effects. A careful analysis shows that the conclusion in [46] related with the quark mass matrices is true; that is, the inclusion of all the one-loop diagrams with the proper Yukawa couplings still leaves the quark mass matrices with determinant equal 
to zero. So, contrary to what is stated in $[42,47]$, the one-loop diagrams are not able by themselves to provide a consistent mass spectrum for the quarks in the context of this economical model. But it does not mean that there is a remanent $U(1)$ symmetry in the full Lagrangian as it is erroneously stated in [46] (in fact, in [47] it is clearly proved that such a $U(1)$ symmetry does not exist at all). The solution to this puzzle and to the controversy raised between $[46,47]$ lies in the two-loop quantum effects which provides a consistent quark mass spectrum via Babu type mechanisms [21]. But this analysis lies outside the scope of this paper and it will be presented elsewhere.

To conclude this section, let us mention that the version of this economical 3-3-1 model developed in the context of the model with right handed neutrinos can be extended to any one of the eight 3 family models presented in Section 4.

\section{Conclusions}

The main motivation of our study was to investigate the neutrino mass spectrum in the framework of the local gauge structure $S U(3)_{c} \otimes S U(3)_{L} \otimes U(1)_{x}$.

To summarize, we have carried out an extensive analysis of the lepton number symmetry in the context of the best known versions of the 3-3-1 model. It is interesting to remark that in one of these versions, namely, the so-called economical model, one explicitly finds the quite unusual situation of the gauging away of the would be Majoron, providing in this way the longitudinal polarization component to a now massive gauge field.

This rare but quite unusual mechanism is related to the fact that the lepton number generator $L$ is connected with the $\lambda_{8}$ generator of $S U(3)_{L}$, as shown in (8).

\section{Conflict of Interests}

The authors declare that there is no conflict of interests regarding the publication of this paper.

\section{Acknowledgments}

The authors thank Enrico Nardi for a written communication and Vicente Vento for his comments. William A. Ponce and Richard H. Benavides thank the "Laboratorio de Física Teórica" from U. de La Plata in Argentina for the warm hospitality during the initial stages of the work which has been partially supported by "Sostenibilidad U. de A. 2014," and "Centro de Investigaciones del ITM."

\section{References}

[1] S. Fukuda, Y. Fukuda, M. Ishitsuka et al., "Constraints on neutrino oscillations using 1258 days of Super-Kamiokande solar neutrino data," Physical Review Letters, vol. 86, no. 25, pp. 5656-5660, 2001.

[2] Q. R. Ahmad, R. C. Allen, T. C. Andersen et al., "Direct evidence for neutrino flavor transformation from neutral-current interactions in the sudbury neutrino observatory," Physical Review Letters, vol. 89, no. 1, Article ID 011301, 6 pages, 2002.
[3] K. Eguchi, S. Enomoto, K. Furuno et al., "First results from KamLAND: evidence for reactor antineutrino disappearance," Physical Review Letters, vol. 90, no. 2, Article ID 021802, 6 pages, 2003.

[4] Y. Ashie, J. Hosaka, K. Ishihara et al., "Evidence for an oscillatory signature in atmospheric neutrino oscillations," Physical Review Letters, vol. 93, Article ID 101801, 2004.

[5] K. Nakamura, K. Hagiwara, K. Hikasa et al., "Review of particle physics," Journal of Physics G, vol. 37, no. 7, Article ID 075021, 2010.

[6] P. Minkowski, " $\mu \rightarrow e \gamma$ at a rate of one out of $10^{9}$ muon decays?" Physics Letters B, vol. 67, no. 4, pp. 421-428, 1977.

[7] M. Gell-Mann, P. Ramond, and R. Slansky, Supergravity, edited by P.van Nieuwenhuizen and D. Z. Freedman, 1980.

[8] T. Yanahida, in Proceedings of the Workshop on the Unified Theory and the Baryon Number in the Universe, O. Sawada and A. Sugamoto, Eds., p. 95, KEK, Tsukuba, Japan, 1979.

[9] R. Mohapatra and G. Senjanović, "Neutrino mass and spontaneous parity nonconservation," Physical Review Letters, vol. 44, no. 14, pp. 912-915, 1980.

[10] R. N. Mohapatra and G. Senjanovic, "Neutrino masses and mixings in gauge models with spontaneous parity violation," Physical Review D, vol. 23, p. 165, 1981.

[11] S. Weinberg, "Baryon- and lepton-nonconserving processes," Physical Review Letters, vol. 43, no. 21, pp. 1566-1570, 1979.

[12] F. Bonnet, M. Hirsch, T. Ota, and W. Winter, "Systematic study of the $d=5$ Wein-berg operator at one-loop order," Journal of High Energy Physics, vol. 2012, no. 7, article 153, 2012.

[13] G. B. Gelmini and M. Roncadelli, "Left-handed neutrino mass scale and spontaneously broken lepton number," Physics Letters $B$, vol. 99 , no. 5 , pp. $411-415,1981$.

[14] J. Steinberger, "First results at the LEP $e^{+} e^{-}$collider," Physics Reports, vol. 203, p. 345, 1991.

[15] E. Accomando, A. Andreazza, H. Anlaufc et al., "Physics with $e^{+} e^{-}$linear colliders," Physics Reports, vol. 299, pp. 1-78, 1998.

[16] M. Günther, J. Hellmig, G. Heusser et al., "Bounds on new Majoron models from the Heidelberg-Moscow experiment," Physical Review D, vol. 54, p. 3641, 1996.

[17] R. Tomas, H. Päs, and J. W. F. Valle, "Generalized bounds on Majoron-neutrino couplings," Physical Review D, vol. 64, no. 9, Article ID 0950005, 7 pages, 2001.

[18] A. Zee, "A theory of lepton number violation and neutrino Majorana masses," Physics Letters B, vol. 93, no. 4, pp. 389-393, 1980.

[19] A. Zee, "Charged scalar field and quantum number violations," Physics Letters B, vol. 161, no. 1-3, pp. 141-145, 1985.

[20] D. Chang and A. Zee, "Radiatively induced neutrino Majorana masses and oscillation," Physical Review D, vol. 61, Article ID 071303R, 2000.

[21] A. Zee, "Quantum numbers of Majorana neutrino masses," Nuclear Physics B, vol. 246, p. 99, 1986.

[22] K. S. Babu, "Model of "calculable" Majorana neutrino masses," Physics Letters B, vol. 203, no. 1-2, pp. 132-136, 1988.

[23] D. Restrepo, O. Zapata, and C. E. Yaguna, "Models with radiative neutrino masses and viable dark matter candidates," Journal of High Energy Physics, vol. 2013, article 11, 2013.

[24] F. Pisano and V. Pleitez, "An $S U(3) \otimes U(1)$ model for electroweak interactions," Physical Review D, vol. 46, p. 410, 1992.

[25] P. Frampton, "Chiral dilepton model and the flavor question," Physical Review Letters, vol. 69, no. 20, pp. 2889-2891, 1992. 
[26] J. T. Liu and D. Ng, "Lepton-flavor-changing processes and CP violation in the $\mathrm{SU}(3)_{c} \times \mathrm{SU}(3)_{L} \times \mathrm{U}(1)_{X}$ model," Physical Review $D$, vol. 50, p. 548, 1994.

[27] M. B. Tully and G. C. Joshi, "Generating neutrino mass in the 33-1 model," Physical Review D, vol. 64, Article ID 011301, 4 pages, 2001.

[28] J. C. Montero, C. A. D. S. Pires, and V. Pleitez, "Comment on 'Majoron emitting neutrinoless double beta decay in the electroweak chiral gauge extensions," Physical Review D, vol. 60, Article ID 098701, 1999.

[29] J. C. Montero, C. A. D. S. Pires, and V. Pleitez, "Spontaneous breaking of a global symmetry in a 3-3-1 model," Physical Review $D$, vol. 60, Article ID 115003, 1999.

[30] L. F. Li, Y. Liu, and L. Wolfenstein, "Hidden higgs particles," Physics Letters B, vol. 159, no. 1, pp. 45-48, 1985.

[31] W. A. Ponce, J. B. Flórez, and L. A. Sánchez, "Analysis of $S U(3)_{c} \times$ $\mathrm{SU}(3)_{L} \times \mathrm{U}(1)_{X}$ local Gauge theory," International Journal of Modern Physics A, vol. 17, p. 643, 2002.

[32] D. L. Anderson and M. Sher, "3-3-1 models with unique lepton generations," Physical Review D, vol. 72, no. 9, Article ID 095014, 9 pages, 2005.

[33] J. C. Montero, F. Pisano, and V. Pleitez, "Neutral currents and Glashow-Iliopoulos-Maiani mechanism in $\mathrm{SU}(3)_{L} \otimes \mathrm{U}(1)_{N}$ models for electroweak interactions," Physical Review D, vol. 47, no. 7, pp. 2918-2929, 1993.

[34] R. Foot, H. N. Long, and T. A. Tran, " $S U(3)_{L} \otimes U(1)_{N}$ and $S U(4)_{L} \otimes U(1)_{N}$ gauge models with right-handed neutrinos," Physical Review D, vol. 50, no. 1, pp. R34-R38, 1994.

[35] D. Chang and H. N. Long, "Interesting radiative patterns of neutrino mass in an $\mathrm{SU}(3)_{C} \otimes \mathrm{SU}(3)_{L} \otimes \mathrm{U}(1)_{X}$ model with righthanded neutrinos," Physical Review D, vol. 73, no. 5, Article ID 053006, 17 pages, 2006.

[36] C. A. S. de Pires and P. S. R. da Silva, "Spontaneous breaking of the lepton number and invisible majoron in a 3-3-1 model," The European Physical Journal C-Particles and Fields, vol. 36, no. 3, pp. 397-403, 2004.

[37] M. Özer, "SU(3) $)_{L} \times \mathrm{U}(1)_{X}$ model of electroweak interactions without exotic quarks," Physical Review D, vol. 54, no. 1, pp. 1143-1149, 1996.

[38] W. A. Ponce and O. Zapata, "Lepton masses and mixing without Yukawa hierarchies," Physical Review D, vol. 74, no. 9, Article ID 093007, 7 pages, 2006.

[39] J. C. Salazar, W. A. Ponce, and D. A. Gutiérrez, "Phenomenology of the $S U(3)_{c} \otimes S U(3)_{L} \otimes U(1)_{X}$ model with exotic charged leptons," Physical Review D, vol. 75, no. 7, Article ID 075016, 17 pages, 2007.

[40] W. A. Ponce, Y. Giraldo, and L. A. Sánchez, "Minimal scalar sector of 3-3-1 models without exotic electric charges," Physical Review D, vol. 67, no. 7, Article ID 075001, 10 pages, 2003.

[41] T. Kitabayashi, "Remark on neutrino masses and oscillations in an $\mathrm{SU}(3)_{L} \times \mathrm{U}(1)_{N}$ model with the radiative mechanism," Physical Review D, vol. 64, no. 5, Article ID 057301, 4 pages, 2001.

[42] P. V. Dong, H. N. Long, D. T. Nhung, and D. V. Soa, "SU(3) $\times$ $\mathrm{SU}(3)_{L} \times \mathrm{U}(1)_{X}$ model with two Higgs triplets," Physical Review $D$, vol. 73, Article ID 035004, 2006.

[43] P. V. Dong, H. N. Long, and D. V. Soa, "Interesting radiative patterns of neutrino mass in an $\mathrm{SU}(3)_{C} \times \mathrm{SU}(3)_{L} \times \mathrm{U}(1)_{X}$ model with right-handed neutrinos," Physical Review D, vol. 73, no. 5, Article ID 053006, 17 pages, 2006.
[44] P. V. Dong, H. N. Long, and D. T. Nhung, "Atomic parity violation in the economical 3-3-1 model," Physics Letters B, vol. 639, no. 5, pp. 527-533, 2006.

[45] D. A. Gutiérrez, W. A. Ponce, and L. A. Sánchez, "Study of the $\mathrm{SU}(3)_{C} \otimes \mathrm{SU}(3)_{L} \otimes \mathrm{U}(1)_{X}$ model with the minimal scalar sector," International Journal of Modern Physics A, vol. 21, no. 10, p. 2217, 2006.

[46] J. C. Montero and B. L. Sánchez-Vega, "Natural Peccei-Quinn symmetry in the 3-3-1 model with a minimal scalar sector," Physical Review D, vol. 84, Article ID 055019, 2011.

[47] P. V. Dong, H. N. Long, and H. T. Hung, "Question of PecceiQuinn symmetry and quark masses in the economical 3-3-1 model," Physical Review D, vol. 86, Article ID 033002, 2012. 

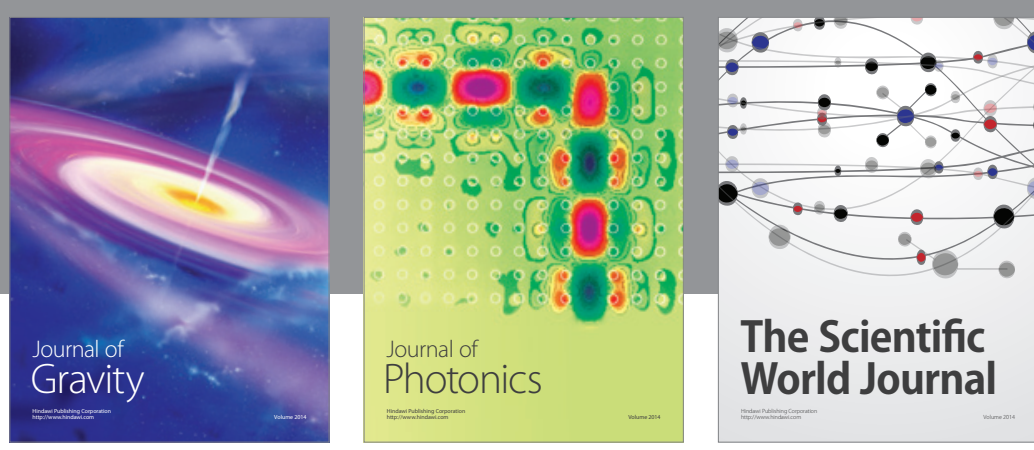

The Scientific World Journal
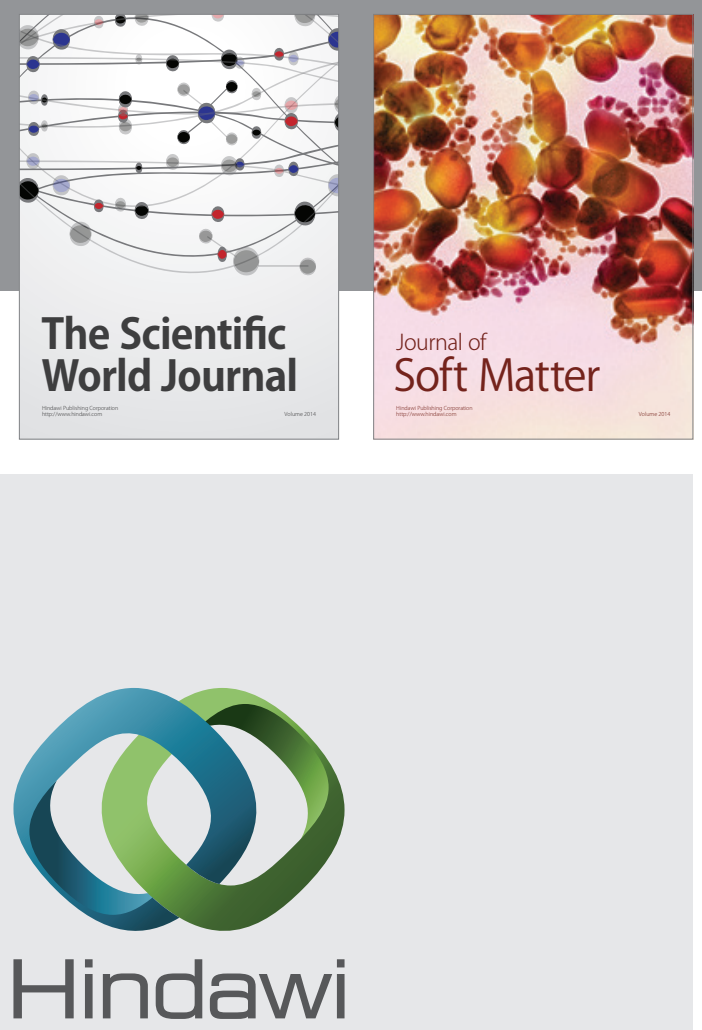

Submit your manuscripts at

http://www.hindawi.com

nternational Journal of

Statistical Mechanics
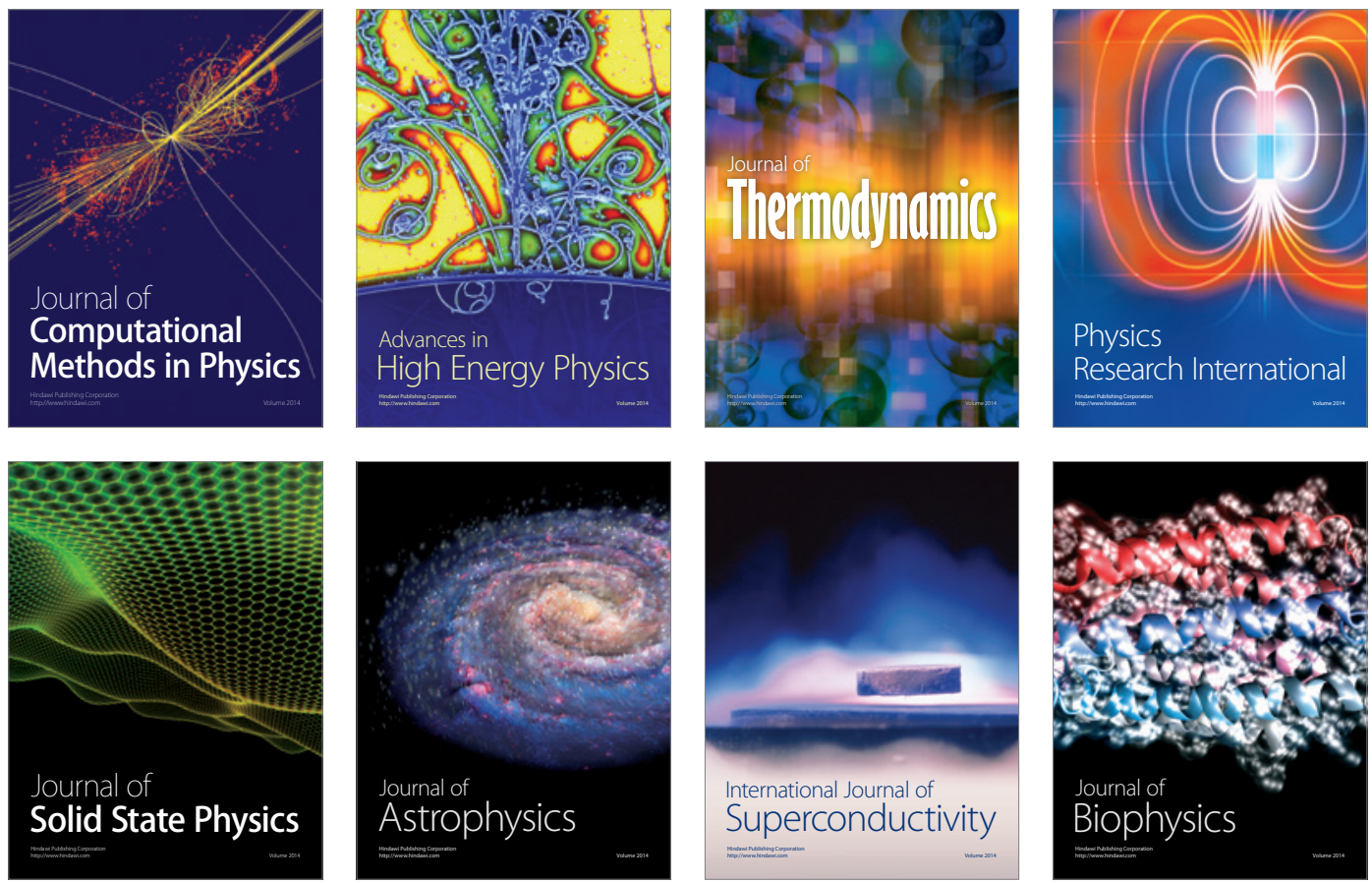
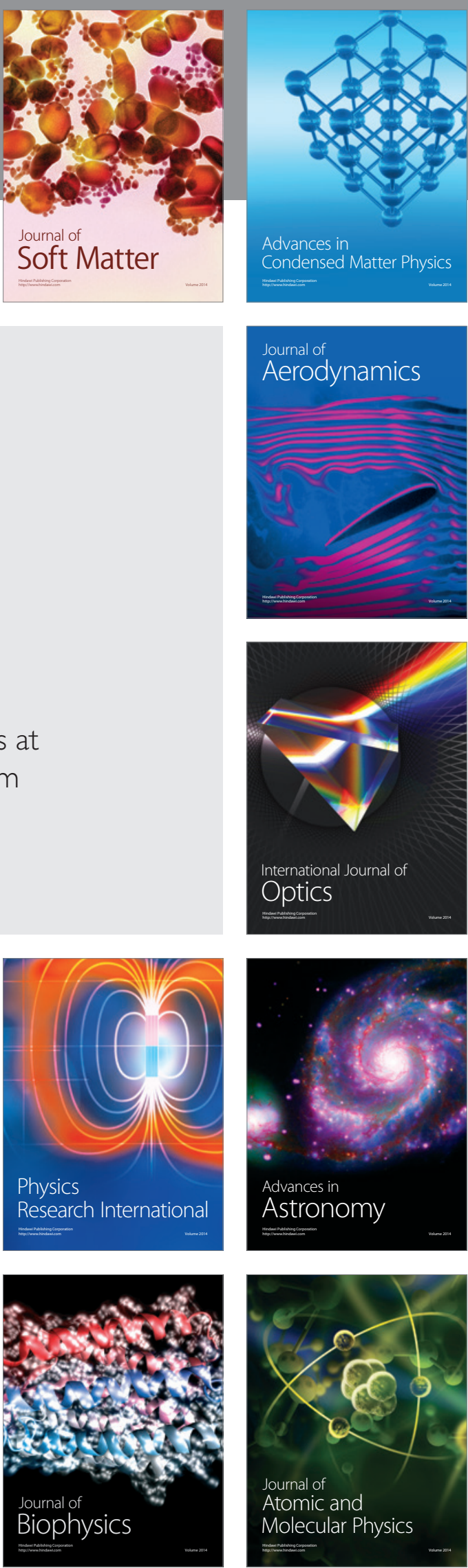